\title{
Hartmann wavefront sensors and their application at FLASH
}

\section{Barbara Keitel, Elke Plönjes, Svea Kreis, Marion Kuhlmann, Kai Tiedtke, Tobias Mey, Bernd Schäfer and Klaus Mann}

J. Synchrotron Rad. (2016). 23, 43-49

\section{IU IUCr Journals CRYSTALLOGRAPHY JOURNALS ONLINE}

Copyright (C) International Union of Crystallography

Author(s) of this paper may load this reprint on their own web site or institutional repository provided that this cover page is retained. Republication of this article or its storage in electronic databases other than as specified above is not permitted without prior permission in writing from the IUCr.

For further information see http://journals.iucr.org/services/authorrights.html 
JOURNAL OF

SYNCHROTRON

RADIATION

ISSN 1600-5775

Received 15 July 2015

Accepted 27 October 2015

Edited by D. Cocco, SLAC National Accelerator Laboratory, USA

Keywords: wavefront; Hartmann sensor; free-electron laser; soft X-rays.

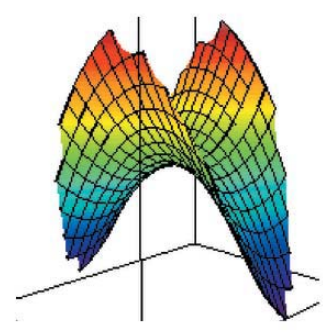

(C) 2016 International Union of Crystallography

\section{Hartmann wavefront sensors and their application at FLASH}

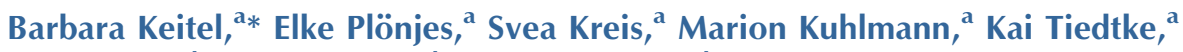 \\ Tobias Mey, ${ }^{\text {b }}$ Bernd Schäfer ${ }^{b}$ and Klaus Mann ${ }^{b}$ \\ ${ }^{a}$ Deutsches Elektronen-Synchrotron DESY, Notkestrasse 85, 22607 Hamburg, Germany, and \\ ${ }^{\mathbf{b}}$ Laser-Laboratorium Göttingen e.V., Hans-Adolf-Krebs-Weg 1, 37077 Göttingen, Germany. \\ *Correspondence e-mail: barbara.keitel@desy.de
}

Different types of Hartmann wavefront sensors are presented which are usable for a variety of applications in the soft X-ray spectral region at FLASH, the freeelectron laser (FEL) in Hamburg. As a typical application, online measurements of photon beam parameters during mirror alignment are reported on. A compact Hartmann sensor, operating in the wavelength range from 4 to $38 \mathrm{~nm}$, was used to determine the wavefront quality as well as aberrations of individual FEL pulses during the alignment procedure. Beam characterization and alignment of the focusing optics of the FLASH beamline BL3 were performed with $\lambda_{13.5 \mathrm{~nm}} / 116$ accuracy for wavefront r.m.s. $\left(w_{\text {rms }}\right)$ repeatability, resulting in a reduction of $w_{\text {rms }}$ by $33 \%$ during alignment.

\section{Introduction}

FLASH, the Free-electron LASer in Hamburg, operates in the soft X-ray spectral region based on the self-amplified spontaneous emission (SASE) process in which laser emission is built up from spontaneous undulator radiation (Ackermann $e t$ al., 2007). Owing to the stochastic nature of this process, the beam characteristics differ from pulse to pulse; thus, requiring single-pulse-resolved photon diagnostics and online characterization of the photon beam parameters (Tiedtke et al., 2009).

Hartmann-Shack and Hartmann sensors are used for realtime wavefront detection and beam characterization in the near infrared, visible and soft X-ray spectral region. They cover nearly the entire wavelength region of FLASH, 4.2$47 \mathrm{~nm}$. FLASH provides intensities up to $250 \mu \mathrm{J}$ per pulse (Plönjes \& Tiedtke, 2015), which is sufficient to measure the intensity distribution (beam profile) and the wavefront (directional distribution) of the radiation field for individual pulses. Under the assumption of a fully coherent beam, these distributions enable the evaluation of paraxial beam parameters such as the beam width $d$, the divergence $\theta$, the beam propagation factor $M^{2}$, the Rayleigh length $z_{\mathrm{R}}$, the waist position $z_{0}$ and the waist diameter $d_{0}$ as defined by Schäfer $\&$ Mann (2002). A prediction of the intensity profile in any desired plane in the direction of propagation can be calculated by solving the Fresnel-Kirchhoff integral (Born \& Wolf, 1985). In this paper, we present the results from measurements at the FLASH beamline BL3 performed with a compact Hartmann sensor jointly developed by Laser-Laboratorium Göttingen (LLG) and DESY (Flöter et al., 2010, 2011). This detector was built for photon diagnostics, beamline alignment and monitoring of free-electron laser (FEL) radiation at FLASH. The determination of beam parameters relevant for many user 
experiments as well as the alignment of the ellipsoidal focusing mirror are described.

\section{Operation principle}

\subsection{Hartmann wavefront sensor}

The wavefront $w\left(x, y ; z_{0}\right)$ of a beam is defined as a surface perpendicular to its local direction of propagation, i.e. perpendicular to the Poynting vector $S(x, y)$ at a position $z_{0}$ on the optical axis (ISO, 2005). Hartmann wavefront measurements are based on the geometric optical determination of the local radiation angles $\left(\beta_{x}, \beta_{y}\right)_{i j}$ by using a pinhole array, the so-called Hartmann plate (Hartmann, 1900), which divides the incoming beam into an array of small sub-beams (see Fig. 1). A camera with a charge-coupled device (CCD) chip is installed at a certain distance $l$ behind this plate and enables the recording of the spot distribution, yielding the precise position of the sub-beams by computing the respective centroids (first moments). The displacement $\Delta x$ of a spot centroid position $\left(x_{\mathrm{c}}, y_{\mathrm{c}}\right)_{i j}$ with respect to the plane wave reference position $\left(x_{\mathrm{r}}, y_{\mathrm{r}}\right)_{i j}$, divided by the distance $l$ yields the local radiation angle $\left(\beta_{x}, \beta_{y}\right)_{i j}$ and, thus, the local wavefront gradient inside one sub-aperture:

$$
\left(\begin{array}{l}
\partial w / \partial x \\
\partial w / \partial y
\end{array}\right)_{i j}=\left(\beta_{x}, \beta_{y}\right)_{i j}=\frac{1}{l}\left(\begin{array}{l}
x_{\mathrm{c}}-x_{\mathrm{r}} \\
y_{\mathrm{c}}-y_{\mathrm{r}}
\end{array}\right)_{i j}
$$

The wavefront is reconstructed from these local gradients using mathematical algorithms. For spherical profiles, a modal expansion according to the works by Cubalchini (1979), Noll (1978) and ISO (2005) in Zernike polynomials is most adequate (Schäfer et al., 2006), because the expansion coefficients correspond to the image aberrations in Seidel's aberration theory (Born \& Wolf, 1985). Summation over all pixel values belonging to the individual sub-apertures samples the

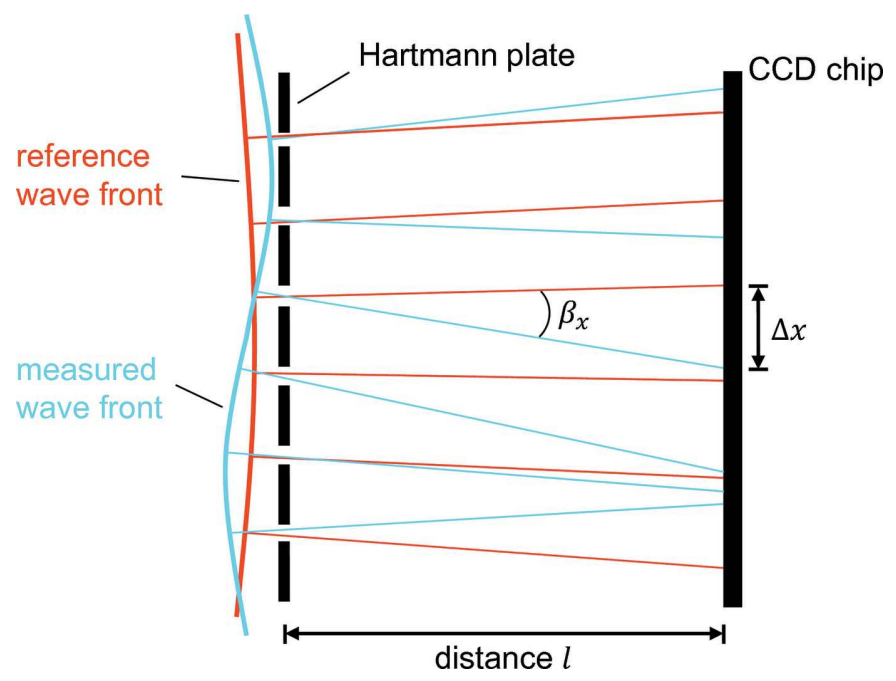

Figure 1

Hartmann principle. The incoming beam (blue) is divided into an array of partial beams by the Hartmann plate. The centroid deviation $\Delta x$ from the known reference spot position divided by the distance $l$ yields the local wavefront gradient $\beta_{x}$ relative to the reference wavefront (red). intensity distribution or beam profile $I(x, y)$. The Hartmann data, consisting of sampled intensity and wavefront gradients $\beta_{x}$, facilitates computation of the first- and second-order moments of the spatial $(x, y)$ and angular $(u, v)$ coordinates over the intensity distribution (Schäfer \& Mann, 2002).

This information is sufficient to compute a number of beam parameters (Schäfer \& Mann, 2002; Schäfer et al., 2006) such as the beam width $d$, the divergence $\theta$, the beam propagation factor $M^{2}$, the waist diameter $d_{0}$, the Rayleigh length $z_{\mathrm{R}}$ and the waist position $z_{0}$ of paraxial beams. This evaluation assumes a fully coherent beam. The computation of secondmoment beam parameters from Hartmann data for soft X-ray radiation and the general agreement with caustic scan techniques (Mey et al., 2014; Mey, 2015) and PMMA imprints were reported by Flöter et al. (2010) and Flöter (2012). Further investigations of wavefront measurements in comparison with PMMA imprints are presented by Dziarzhytski et al. (2016). Once intensity and phase of the beam are known from the Hartmann measurements, a Fresnel-Kirchhoff propagation yields the intensity distribution at different $z$ positions (Born \& Wolf, 1985).

The area of interest (AOI) for the wavefront and the beam profile evaluation is selected by clipping noise at a level of $1 \%$ of the full dynamic range of the CCD camera. The evaluation radius $a$ is defined by the largest circle inscribed into this AOI. The quality of the wavefront is expressed in terms of the wavefront peak-to-valley $w_{\mathrm{PV}}$ and the wavefront root-meansquare $w_{\text {rms }}$ values. Both are calculated after subtraction of tip/tilt and defocus terms.

The software MrBeam (Laser-Laboratorium Göttingen, http://llg-ev.de) allows the selection of the appropriate polynomial basis (either Zernike in the case of circular beams or Legendre for rectangular beams) for wavefront reconstruction and the desired degree of expansion. For Zernike polynomials either the degree $n$ according to Born and Wolf notation or the 37 polynomials of the Arizona fringe set can be chosen. The reconstruction mode can also be selected: the modal technique uses the polynomial basis (see above) whereas the zonal approach utilizes a finite difference approximation of the measured local gradients (Malacara, 2007).

\subsection{The reference wavefront}

The wavefront evaluation is carried out by using a comparison of the measured wavefront to a reference wavefront, typically either a plane or a spherical wave. In the case of the compact Hartmann sensor (see $\$ 3.1$ ), a $5 \mu \mathrm{m}$ pinhole was placed into the FEL beam to generate a spherical wavefront (Mercère et al., 2003). The pinhole was located in the focused beam of beamline BL2, $110 \mathrm{~mm}$ upstream of the focal plane. The Hartmann plate is placed into a central Airy disk at a distance measured to be $3592 \mathrm{~mm}$ behind the pinhole (for $13.5 \mathrm{~nm}$ ). A FEL pulse train of 30 bunches at a wavelength of $13.5 \mathrm{~nm}$ was used for the calibration measurement. A singlepulse energy of about $80 \mu \mathrm{J}$ was attenuated using a $216 \mathrm{~nm}$ thick Si filter foil, without a supporting mesh, to prevent destruction of the pinhole by the FLASH photon beam. To 


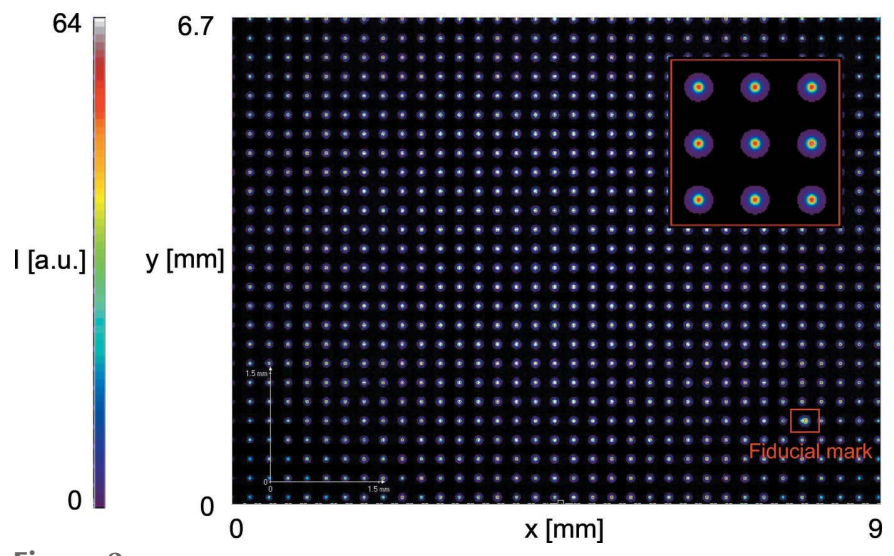

Figure 2

Compact Hartmann sensor: a reference spot pattern is created by diffraction from a $5 \mu \mathrm{m}$ pinhole. For alignment purposes, one pinhole (Fiducial mark) bottom right on the Hartmann plate shows a distinct size and shape. It defines the sensor orientation and helps with the identification of individual sub-apertures.

obtain an improved signal-to-noise ratio, 100 camera frames, each containing a pulse train, were averaged to create the reference shown in Fig. 2. The beam profile was centered on the wavefront sensor with the optical axis perpendicular to the CCD chip. A slightly bigger pinhole (see right bottom in Fig. 2) in the Hartmann plate defines the sensor orientation to the beam. For all other available wavefront sensors at FLASH, reference wavefronts were measured under similar conditions.

\subsection{Wavelength range and resolution}

For a short wavelength, the nickel foils used for the pinhole array became transparent (Flöter, 2012), thus defining the short-wavelength limit for the wavefront sensor. In addition, the number of illuminated pixels of each sub-beam on the CCD camera becomes too small for computing the respective centroid. Diffraction on the sub-apertures (diameter $d$ ) of the Hartmann plate defines the upper wavelength $\lambda$ which can be estimated with the aid of an Airy disk (Born \& Wolf, 1985; Hecht, 2001). Far away from the sub-aperture, the diffraction angle $\theta$ at which the first minimum occurs around the central maximum, is given for small angles by $\theta \approx 1.22(\lambda / d)$. Two spots from the Hartmann plate can still be resolved when the maximum of the first Airy pattern falls on top of the first minimum of the second Airy pattern (Rayleigh criterion). For a distance $l$ between the CCD and the Hartmann plate and if the diameter of the Airy disk on the CCD is less than the pinhole pitch $p$, the following formula results for the wavelength: $\lambda \leq(p d) /(2.44 l)$.

In the following sections, the resolution of the different wavefront sensors is given as the experimentally determined wavefront repeatability.

\section{Available wavefront sensors at FLASH}

\subsection{Compact Hartmann sensor}

The compact Hartmann sensor (see Fig. 3) was jointly developed by LLG and DESY. The setup consists of a pinhole array and a camera with a 14-bit dynamic range (Softhard SHT MR285MC) with a charge-coupled device (CCD) chip at a distance $l=198.3 \mathrm{~mm}$ behind the pinhole array. The distance represents a compromise between attainable wavefront sensitivity at short wavelengths and spatial resolution at long wavelengths, as described in $\$ 2.1$. The wavelength range of the compact Hartmann sensor is 4-38 nm (see \$2.3).

The CCD chip consists of $1392 \times 1040$ pixels with a $6.45 \mu \mathrm{m}$ $\times 6.45 \mu \mathrm{m}$ pixel size, resulting in a field of view of $8.9 \mathrm{~mm} \times$ $6.7 \mathrm{~mm}$. It is coated with a phosphor $\left(3-4 \mu \mathrm{m} \mathrm{Gd}_{2} \mathrm{O}_{2} \mathrm{~S}: \mathrm{Tb}\right.$, grain size $1-2 \mu \mathrm{m}$, central emission wavelength $545 \mathrm{~nm}$ ) for converting the soft X-rays into visible light. The Hartmann plate is a pinhole array, made of a $20 \mu \mathrm{m}$-thick nickel foil with electroformed holes (diameter $d=75 \mu \mathrm{m}$ and pitch $p=$ $250 \mu \mathrm{m}$ ) produced by Precision Eforming LLC (http:// www.precisioneforming.com/). To adapt the Hartmann sensor to other wavelength ranges, the plate is exchangeable. However, this requires the generation of a new reference wavefront (see \$2.2).

The compact Hartmann sensor operates at $10 \mathrm{~Hz}$ using the external trigger signal provided from a chopper integrated into the FLASH beamlines. Compared with our very compact, self-supporting Hartmann sensor (see $\$ 3.2$ and Flöter et al., 2010), this sensor shows a higher mechanical stability and plane parallelism owing to a rigid setup between CCD and Hartmann plate holder.

The average relative single-pulse repeatability of the wavefront measurements was determined against the reference shown in Fig. 2. A series of 100 single FEL pulses (1 bunch per train) was recorded behind the $5 \mu \mathrm{m}$ pinhole at $\lambda=13.5 \mathrm{~nm}$. Using an evaluation radius for the Zernike reconstruction of $a=3.07 \mathrm{~mm}$ ( $\$ 2.1)$, a mean deviation for the peak-to-valley value $w_{\mathrm{PV}}$ of $\lambda / 19$ and for the root-mean-square value $w_{\text {rms }}$ of $\lambda / 116$ was calculated.
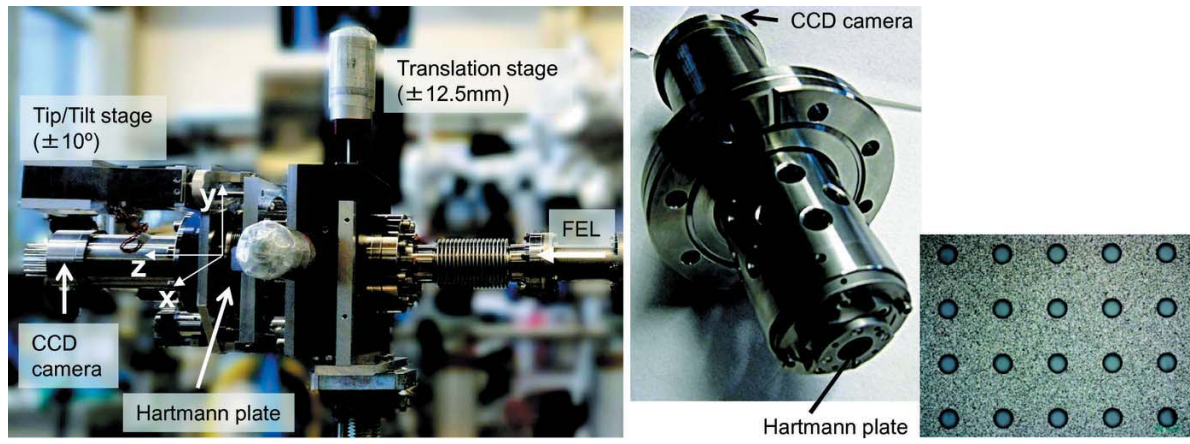

Figure 3

Left: compact Hartmann sensor. A manual operating stage allows for lateral movement in the $x$ and $y$ direction of $\pm 12.5 \mathrm{~mm}$ and $\pm 10^{\circ}$ tip/tilt to align the sensor to the beam. The sensor is mounted on a tripod and connected to the beamline or user experiment using a CF40 bellow. Right: inner part of the Hartmann sensor with the Hartmann plate holder. In the inset an optical microscopic image (magnification 10x) of part of the nickel Hartmann plate with electroformed holes is shown. 


\subsection{Very compact, self-supporting Hartmann sensor}

The first version of a Hartmann sensor (see Fig. 4), developed in collaboration with LLG, is a very compact design which is used in the wavelength range 4-35 $\mathrm{nm}$. The smaller distance between the Hartmann plate and the CCD of $l=97 \mathrm{~mm}$ also permits measuring at longer wavelengths of up to $80 \mathrm{~nm}$ (see $\$ 2.3$ ). Owing to its low weight this sensor is self-supporting and can be mounted directly on a user's experimental chambers using a CF40 tube section. However, the short distance between the Hartmann plate and the CCD of $97 \mathrm{~mm}$ and the lower mechanical stability limits the obtainable resolution of the system to $\lambda / 41$ for the wavefront repeatability $w_{\text {rms }}$. The resolution is about a factor of three less compared with the compact Hartmann wavefront sensor (see $\$ 3.1$ and Flöter, 2012). The Hartmann plate is again made of a $20 \mu \mathrm{m}$-thick nickel foil with electroformed holes (diameter $d=$ $75 \mu \mathrm{m}$ and pitch $p=250 \mu \mathrm{m}$ ) by Precision Eforming LLC. This early design does not incorporate a specific alignment pinhole in its Hartmann plate.

In this detector, a 12-bit dynamic range $\mathrm{CCD}$ camera (Lumenera LM165M) with $1280 \times 1024$ pixels is used which can be operated at $10 \mathrm{~Hz}$. The field of view is $8.25 \mathrm{~mm} \times$ $6.6 \mathrm{~mm}$ with $6.45 \mu \mathrm{m} \times 6.45 \mu \mathrm{m}$ pixel size. For converting the soft X-rays into visible light, the CCD chip is coated with a phosphor (3-4 $\mu \mathrm{m} \quad \mathrm{Gd}_{2} \mathrm{O}_{2} \mathrm{~S}: \mathrm{Tb}$, grain size 1-2 $\mu \mathrm{m}$, central emission wavelength $545 \mathrm{~nm}$ ). A manual operating stage allows for lateral movement in the $x$ - and $y$-directions of $\pm 10 \mathrm{~mm}$ and a tip/tilt movement of $\pm 10^{\circ}$.

As described in \$3.1, the average relative single-pulse repeatability was determined. Using an evaluation radius of $a=3.07 \mathrm{~mm}$, a mean deviation for the peak-to-valley value $w_{\mathrm{PV}}$ of $\lambda / 9$ and for the root-mean-square value $w_{\text {rms }}$ of $\lambda / 41$ was calculated for $\lambda=13.5 \mathrm{~nm}$. Tip/tilt and defocus terms were subtracted prior to the computation of the $w_{\mathrm{PV}}$ and the $w_{\text {rms }}$ values (Flöter, 2012).

Using a Hartmann plate made of $7 \mu \mathrm{m}$ tantalum foil with circular laser-drilled holes ( $65 \mu \mathrm{m}$ diameter, $320 \mu \mathrm{m}$ pitch), the wavelength range could be extended down to $1 \mathrm{~nm}$. The achieved accuracy at $\lambda=13.5 \mathrm{~nm}$ is about $\lambda / 90$ for $w_{\text {rms }}$ and $\lambda / 15$ for $w_{\mathrm{PV}}$ (Flöter, 2012).

\subsection{Large field of view wavefront sensor}

The hardware of our large field of view wavefront sensor was manufactured by the company Imagine Optic SA (http:// www.imagine-optic.com/en/). A representative setup is shown in Fig. 5. This sensor is typically used in the spectral region of 10-40 nm and uses a 16-bit dynamic range back-illuminated CCD camera of type PI-SX:1300 from Princeton Instruments.
According to the estimation in $\$ 2.3$, the sensor is usable for wavelengths up to $70 \mathrm{~nm}$. The CCD camera has $1340 \times 1300$ pixels with a $20 \mu \mathrm{m} \times 20 \mu \mathrm{m}$ pixel size resulting in a large field of view of $19.5 \mathrm{~mm} \times 19.5 \mathrm{~mm}$. This is a big advantage for investigating unfocused or strongly divergent beams or enabling setup behind user experiments at a larger distance to the focal plane. The back-illuminated CCD camera also allows the characterization of low-intensity beams. The camera operates at $0.45 \mathrm{~Hz}$ using the external trigger signal provided from a chopper integrated into the FLASH beamlines.

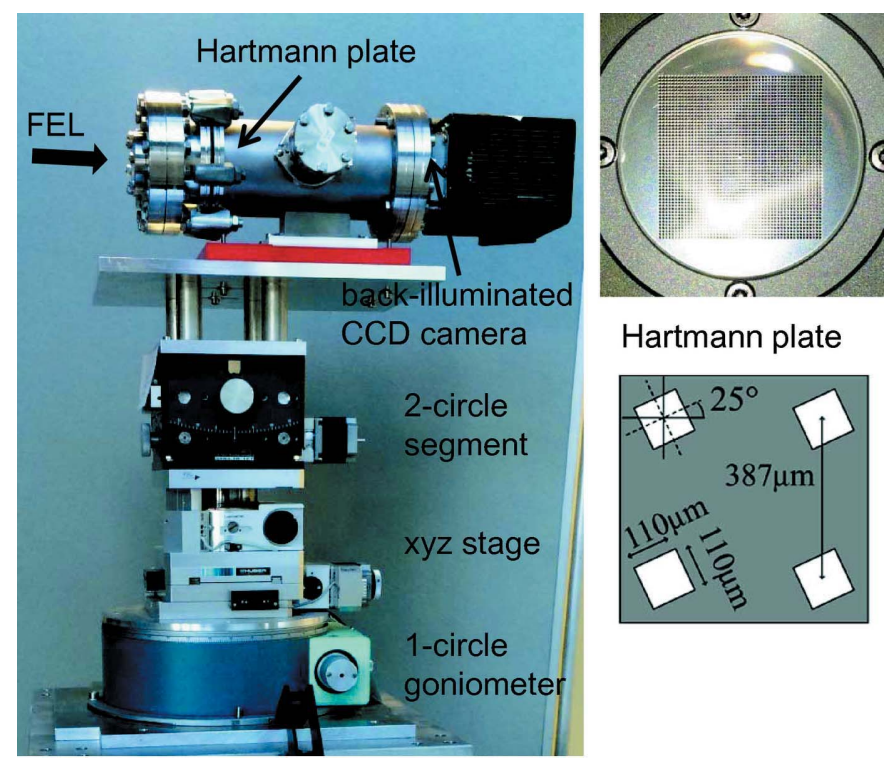

Figure 5

Left: large field of view wavefront sensor. It is connected to the beamline or a user experiment using a CF40 bellow. The alignment of the sensor to the beam is realised by a six-axis stacked movement unit. Right: the nickel Hartmann plate consist of squared holes each tilted by $25^{\circ}$. The central hole of the plate is omitted for alignment purposes. 
The Hartmann plate, mounted at a distance of $250 \mathrm{~mm}$ in front of the CCD camera, is manufactured of a $80 \mu \mathrm{m}$ nickel plate with $51 \times 51$ quadratic holes each tilted by $25^{\circ}$ (see Fig. 5). The holes are $110 \mu \mathrm{m} \times 110 \mu \mathrm{m}$ in size with a pitch of $387 \mu \mathrm{m}$. Due to this design, interference of adjacent holes is prevented and, therefore, the spatial resolution is increased and a high dynamic range is achieved (Mercère et al., 2003). The wavefront repeatability at $\lambda=13.4 \mathrm{~nm}$ is specified to $\lambda / 100$ for $w_{\text {rms }}$. In this Hartmann plate, the central hole is omitted for alignment purposes, defining the correct sensor orientation and allowing for individual sub-apertures to be identified (see Fig. 5).

The large field of view Hartmann sensor requires a six-axis stacked movement unit for alignment as shown in Fig. 5; owing to its size and heavy weight it is less flexible in setup but it achieves a higher measurement accuracy and allows investigation with low-intensity radiation due to its more sensitive camera.

\subsection{Software}

All presented wavefront sensors, including the one from Imagine Optic, are operated using the software MrBeam which allows for the online calculation of wavefront, beam parameters and aberrations. Also the computation of backpropagated beam profiles to any position along the propagation axis is possible.

\subsection{Outlook: compact Hartmann sensor with larger field of view}

A compact Hartmann wavefront sensor with a larger field of view is currently developed jointly by LLG and DESY. It will allow for investigations of the unfocused beam at FLASH or highly divergent beams after e.g. Kirkpatrick-Baez-optics systems. A CCD camera from Softhard with $2048 \times 2048$ pixels and a $7.4 \mu \mathrm{m} \times 7.4 \mu \mathrm{m}$ pixel size results in a sensitive detector area of $15.2 \mathrm{~mm} \times 15.2 \mathrm{~mm}$. Due to the enlarged field of view the readout time is increased and limits operation at $5 \mathrm{~Hz}$. The Hartmann plate, featuring the same hole diameter and pitch as described above for the compact Hartmann sensor (see §3.1), is $22 \mathrm{~mm}$ in diameter and it is mounted at a distance $l$ of $\sim 200 \mathrm{~mm}$ to the CCD camera. In addition, it is envisaged to have two different Hartmann plates. One plate will operate in the wavelength range between 4 and $38 \mathrm{~nm}$ as described in $\$ 3.1$ and the second plate extends the measurable wavelength range up to $106 \mathrm{~nm}$ using a hole diameter of $d=$ $130 \mu \mathrm{m}$ and a pitch of $p=400 \mu \mathrm{m}$ (see $\$ 2.3$ ). For $x / y$ and tip/tilt alignment of the sensor a manual adjustment unit is provided.

\section{Beamline commissioning at BL3}

The measurements were performed using a wavelength of $13.3 \mathrm{~nm}$ in single-bunch mode with a bunch charge of $0.5 \mathrm{nC}$. The compact Hartmann sensor (see \$3.1) was placed about $850 \mathrm{~mm}$ downstream of the expected focal plane at FLASH beamline BL3 (see Fig. 6) to adapt the beam size of the

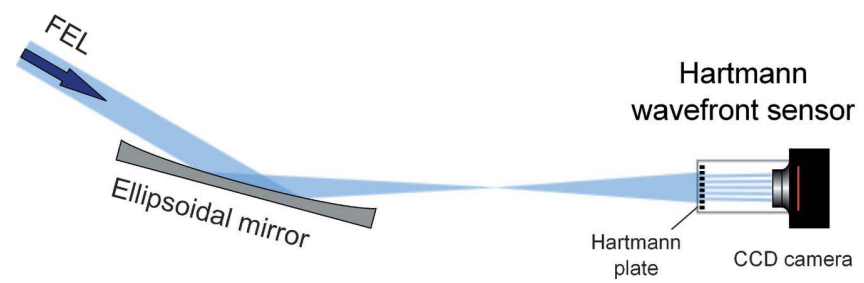

Figure 6

Setup used for the online adjustment of the ellipsoidal grazing-incidence focusing mirror ( $2 \mathrm{~m}$ focal length) of beamline BL3 at FLASH. The ellipsoidal mirror is located at a distance of $72.2 \mathrm{~m}$ from the source.

diverging beam to the sensitive detector area. Two $10 \mathrm{~mm}$ apertures at an upstream distance of about $48.3 \mathrm{~m}$ and $52.8 \mathrm{~m}$ from the focusing ellipsoidal mirror were used. The ellipsoidal mirror was mounted at a $3^{\circ}$ grazing incidence angle, has a focal length of $2 \mathrm{~m}$ and is coated with amorphous carbon (Tiedtke $e t$ $a l ., 2009)$. The intensity of about $65 \mu \mathrm{J}$ was attenuated to about $2 \mu \mathrm{J}$ per pulse using $0.01 \mathrm{mbar} \mathrm{Xe}$ in the gas attenuator (Hahn \& Tiedtke, 2007); the use of foil filters for further attenuation was not required. The camera of the wavefront sensor was triggered by the fast shutter which allowed us to measure the changes of the Zernike coefficients, $w_{\mathrm{rms}}, w_{\mathrm{PV}}$ and further beam parameters during the mirror movement at a $10 \mathrm{~Hz}$ repetition rate.

The focusing mirror of beamline BL3 was aligned by successively moving the mirror with respect to the yaw and pitch angles around a pre-aligned position. The roll angle is not adjustable due to the special mechanical setup of the mirror chamber. The goal of the alignment procedure with the wavefront sensor was to maximize the Strehl value, which is, according to the Maréchal formula Strehl $\approx 1-\left[(2 \pi / \lambda) w_{\text {rms }}\right]^{2}$, equivalent to minimizing $w_{\text {rms }}$ (Born \& Wolf, 1985; Maréchal, 1947). In parallel to the mirror movement the astigmatism Zernike coefficients were calculated online to retain information on the success of the alignment.

First, the yaw angle was aligned such that the astigmatism $x y$ (Zernike coefficient No. 4) was oriented along the vertical and horizontal coordinate axis. Therefore, starting at a mirror yaw angle of $0 \mathrm{mrad}$, we scanned in a range between -0.5 and $1.5 \mathrm{mrad}$. Afterwards, the pitch angle was adjusted in a range of 0.3 and $-1.3 \mathrm{mrad}$ to change the astigmatism $y^{2}-x^{2}$ (Zernike coefficient No. 5). It nearly vanished for a pitch angle of about -1 mrad. Keeping the pitch constant we doublechecked the wavefront while again changing the yaw angle. Only the Zernike astigmatism coefficients respective of the used movement changed significantly during the alignment.

For a yaw angle of $0.65 \mathrm{mrad}$ we again optimized the pitch angle in a fine-adjustment to minimize the $w_{\text {rms }}$ value, finally leading to a reduction of the $w_{\text {rms }}$ value by $68 \%$ from 4 to $1.3 \mathrm{~nm}$ and of $w_{\mathrm{PV}}$ by $56 \%$ from $27 \mathrm{~nm}$ to $12 \mathrm{~nm}$ for a pitch angle of $-0.94 \mathrm{mrad}$. During this alignment procedure no significant change of the Zernike coefficients coma $x$ and coma $y$ was seen. They are one to three orders of magnitude smaller compared with the astigmatism coefficients.

In Fig. 7, wavefronts (evaluation radius $a=2.38 \mathrm{~mm}$ ) measured at the start of the alignment as well as during the 
Table 1

Beam parameter and Zernike coefficients for different steps of the alignment.

For the start of the alignment and during the alignment, single-pulse parameters are given. For the final mirror position parameters are calculated for an average of 100 single pulses. Beam and waist diameter are given in $4 \sigma$. BPF is beam propagation factor.

\begin{tabular}{|c|c|c|c|c|c|c|}
\hline & \multicolumn{2}{|c|}{$\begin{array}{l}\text { Second-moment } \\
\text { beam parameters } \\
\text { at start of the } \\
\text { alignment }\end{array}$} & \multicolumn{2}{|c|}{$\begin{array}{l}\text { Second-moment } \\
\text { beam parameters } \\
\text { during the } \\
\text { alignment }\end{array}$} & \multicolumn{2}{|c|}{$\begin{array}{l}\text { Second-moment } \\
\text { beam parameters } \\
\text { at the final } \\
\text { position }\end{array}$} \\
\hline & $x$ & $y$ & $x$ & $y$ & $x$ & $y$ \\
\hline$w_{\mathrm{PV}}(\mathrm{nm})$ & \multicolumn{2}{|c|}{324.43} & \multicolumn{2}{|c|}{27.24} & \multicolumn{2}{|c|}{12.19} \\
\hline$w_{\mathrm{rms}}(\mathrm{nm})$ & \multicolumn{2}{|c|}{-} & \multicolumn{2}{|c|}{3.97} & \multirow{2}{*}{\multicolumn{2}{|c|}{$\begin{array}{l}1.31 \\
1.82\end{array}$}} \\
\hline $\mathrm{BPF} M^{2}$ & \multicolumn{2}{|c|}{ - } & \multicolumn{2}{|c|}{1.94} & & \\
\hline BPF $M_{i}^{2}$ & - & - & 2.25 & 1.97 & 1.99 & 1.83 \\
\hline Beam diameter $d(\mathrm{~mm})$ & 3.64 & 3.97 & 3.86 & 4.06 & 3.34 & 3.85 \\
\hline Waist position $z_{0}(\mathrm{~mm})$ & -882.85 & -813.87 & -846.63 & -852.61 & -848.94 & -848.63 \\
\hline Waist diameter $d_{0}(\mu \mathrm{m})$ & 107.98 & 90.37 & 8.8 & 7.4 & 9.1 & 7.2 \\
\hline Astigmatism $y^{2}-x^{2}(\mu \mathrm{m})$ & \multicolumn{2}{|c|}{-0.1338} & \multicolumn{2}{|c|}{0.0116} & \multicolumn{2}{|c|}{-0.00113} \\
\hline Astigmatism $x y(\mu \mathrm{m})$ & \multicolumn{2}{|c|}{0.8632} & \multicolumn{2}{|c|}{0.0034} & \multicolumn{2}{|c|}{-0.00145} \\
\hline
\end{tabular}

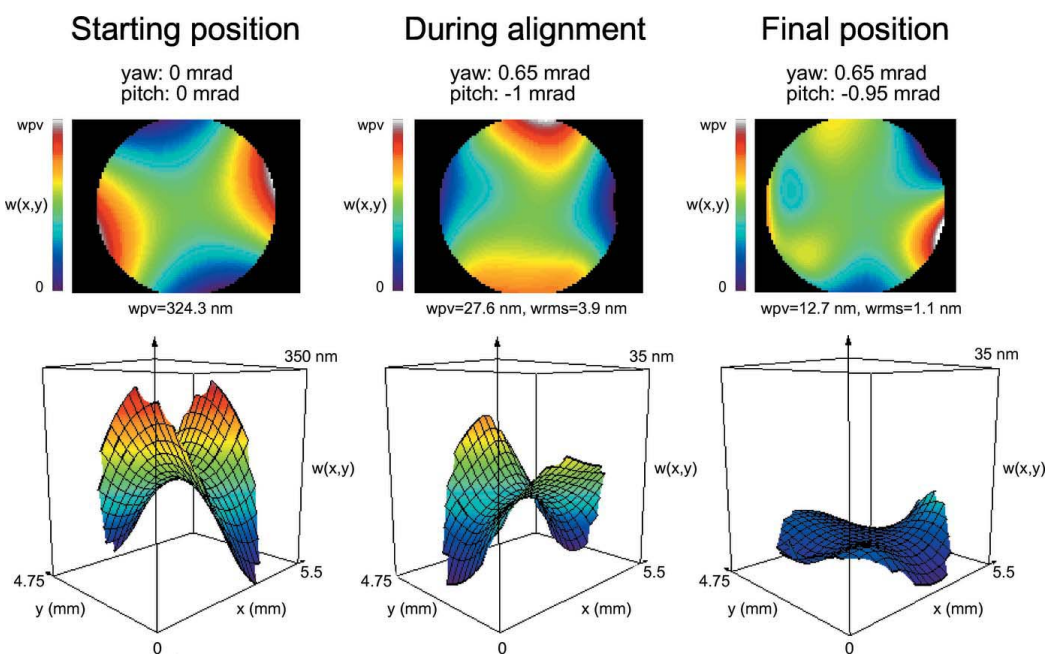

Figure 7

Wavefronts measured at different steps of the alignment procedure of the ellipsoidal focusing mirror at FLASH beamline BL3. The measurements are performed at a wavelength of $13.3 \mathrm{~nm}$, in single-bunch mode and with use of the gas attenuator for intensity reduction. Note that the scale $w(x / y)$ is enlarged by a factor of ten for the figure of the starting position.

alignment procedure and for the final mirror position are shown. The wavefronts shown for the starting conditions and during the alignment are single-pulse measurements whereas the wavefront for the final alignment shows an average of 100 single frames on the CCD chip. In Table 1, the evaluated beam parameters and astigmatism Zernike aberrations are summarized. The Strehl ratio and the focal spot quality are improved accordingly (see Table 1). The final waist position is determined to be $848.8 \mathrm{~mm}$ upstream of the Hartmann plate. From this and the measured distance between the Hartmann plate and the last beamline valve, we calculated the focus position to be at a distance of $528.7 \mathrm{~mm}$ from the flange of the last beamline valve.

The Hartmann data recorded before and after the alignment procedure were used to compute beam profiles close to the waist of the FEL beam by numerical propagation via the Kirchhoff-Fresnel integral (Flöter et al., 2010). The corresponding simulated beam profiles are displayed in Fig. 8. Before the alignment (upper profiles) a strong wavefront astigmatism leads to a change of the profile orientation before and after the focus, whereas after the alignment a more circular beam profile in all planes is observed (lower profiles).

\section{Conclusion}

In this article, we reported on beam characterization measurements for the freeelectron laser FLASH using a Hartmann wavefront sensor for the soft X-ray spectral region with advanced stability and accuracy. The compact device is mounted on a CF63

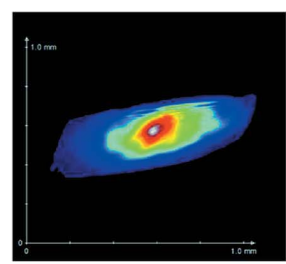

$100 \mathrm{~mm}$ downstream
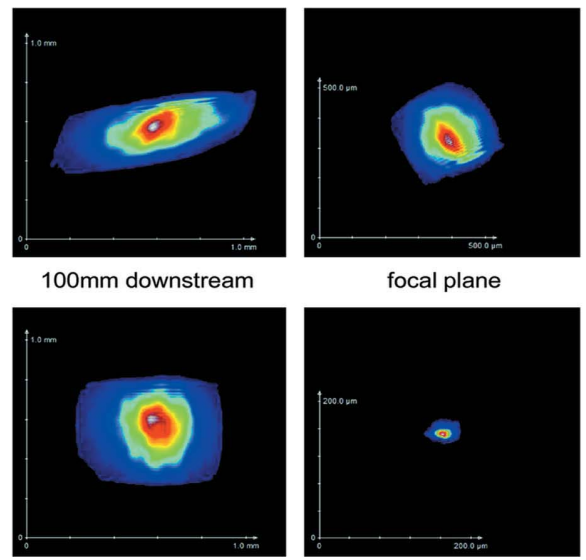

focal plane
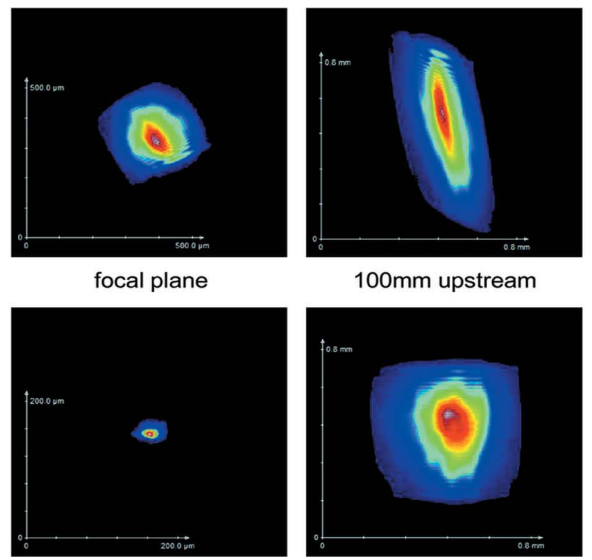

$100 \mathrm{~mm}$ upstream

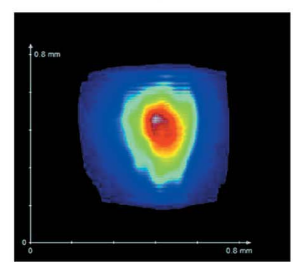

Figure 8

Beam profiles computed for $13 \mathrm{~nm}$ wavelength in the near-waist region ( $\pm 100 \mathrm{~mm}$ around the focal plane) by numerical propagation of the Hartmann data before (top) and after (bottom) the alignment procedure. vacuum flange and can easily be attached to different beamlines or experimental chambers. This Hartmann sensor with high accuracy is now available for FLASH users and can be applied for evaluation of wavefront, Zernike coefficients, intensity profiles and further beam parameters. A single-pulse wavefront repeatability (r.m.s.) was determined against a spherical reference wavefront, corresponding to $\lambda / 116$ at $\lambda=$ $13.5 \mathrm{~nm}$. The device is regularly used for beamline alignment at FLASH as described for the ellipsoidal focusing mirror of beamline BL3. The astigmatic distortion dominating the wavefront before the adjustment could be reduced by over $60 \%$ to $\lambda / 10\left(w_{\text {rms }}\right)$ at $13.3 \mathrm{~nm}$.

We briefly presented two additional wavefront sensors available for users at FLASH, a very compact self-supporting as well as a large field of view version, and described our newly developed compact Hartmann sensor with a bigger field of view currently under development. 


\section{Acknowledgements}

Support of the FLASH user facility, in particular the funding of wavefront sensors through the BMBF program FSP301FLASH, is greatly acknowledged. This work is partly supported by 'IRUVX-PP', EU co-funded project under FP7 (Grant Agreement 211285). We also acknowledge the support from Deutsche Forschungsgemeinschaft within SFB755 'Nanoscale Photonic Imaging'.

\section{References}

Ackermann, W. et al. (2007). Nat. Photon. 1, 336-342.

Born, M. \& Wolf, E. (1985). Principles of Optics, 6th ed. Cambridge University Press.

Cubalchini, R. (1979). J. Opt. Soc. Am. 69, 972-977.

Dziarzhytski, S., Gerasimova, N., Goderich, R., Mey, T., Reininger, R., Rübhausen, M., Siewert, F., Weigelt, H. \& Brenner, G. (2016). J. Synchrotron Rad. 23, 123-131.

Flöter, B., Juranić, P., Großmann, P., Kapitzki, S., Keitel, B., Mann, K., Plönjes, E., Schäfer, B. \& Tiedtke, K. (2011). Nucl. Instrum. Methods A, 635, 108-112.

Flöter, B. (2012). Strahlcharakterisierung von Freie-ElektronenLasern im weichen Röntgen-Spektralbereich, Göttingen Series in $X$-ray Physics, Universitätsverlag Göttingen, Göttingen, Germany (http://webdoc.sub.gwdg.de/univerlag/2012/GSXP8_floeter.pdf).
Flöter, B., Juranić, P., Kapitzki, S., Keitel, B., Mann, K., Plönjes, E., Schäfer, B. \& Tiedtke, K. (2010). New J. Phys. 12, 083015.

Hahn, U. \& Tiedtke, K. (2007). AIP Conf. Proc. 879, 276-282.

Hartmann, J. (1900). Z. Instrum. 20, 47-58.

Hecht, E. (2001). Optics, 4th ed. Reading: Addison Wesley.

ISO (2005). ISO 15367-2:2005. Laser and laser-related equipment Test methods for determination of the shape of a laser beam wavefront - Part 2: Shack-Hartmann sensors. http://www.iso.org/ iso/catalogue_detail.htm?csnumber $=33629$.

Malacara, D. (2007). Optical Shop Testing, 3th ed. New Jersey: John Wiley and Sons.

Maréchal, A. (1947). Rev. Opt. 26, 257.

Mercère, P., Zeitoun, P., Idir, M., Le Pape, S., Douillet, D., Levecq, X., Dovillaire, G., Bucourt, S., Goldberg, K. A., Naulleau, P. P. \& Rekawa, S. (2003). Opt. Lett. 28, 1534-1536.

Mey, T. (2015). Radiation characteristics of extreme $U V$ and soft $X$-ray sources, Göttingen Series in X-ray Physics, Vol. 15. Universitätsverlag Göttingen, Göttingen, Germany (http://resolver.sub.unigoettingen.de/purl? univerlag-isbn-978-3-86395-211-2).

Mey, T., Schäfer, B., Mann, K., Keitel, B., Kuhlmann, M. \& Plönjes, E. (2014). Opt. Express, 22, 16571-16584.

Noll, J. R. (1978). J. Opt. Soc. Am. 68, 139-140.

Plönjes, E. \& Tiedtke, K. (2015). Optical Technologies for ExtremeUltraviolet and Soft X-ray Coherent Sources, edited by F. Canova and L. Poletto, Springer Series in Optical Sciences, Vol. 197.

Schäfer, B., Lübbecke, M. \& Mann, K. (2006). Rev. Sci. Instrum. 77, 053103.

Schäfer, B. \& Mann, K. (2002). Appl. Opt. 41, 2809-2817.

Tiedtke, K. et al. (2009). New J. Phys. 11, 023029. 